\title{
The Machining Error Compensation Method for Aeronautical Thin-walled Parts Machining Process
}

\author{
Zhao Ju${ }^{1}$, Chen Bing ${ }^{1}$, Yang Jie ${ }^{1}$, Yang Qing-long ${ }^{1}$ \\ ${ }^{1}$ Key Laboratory of Contemporary Design and Integrated Manufacturing Technology, Ministry of \\ Education, Northwestern Polytechnical University, Shannxi Xi'an, 710072,China
}

\begin{abstract}
Keywords: Thin-walled parts; Machining Error; Error model; Multivariate Engineering Process Control(MEPC); Anti Deformation Compensation
\end{abstract}

\begin{abstract}
A machining error compensation method for Aeronautical thin-walled parts machining process is proposed to improve the processing quality, reduce rework times and improve processing efficiency. Based on the analysis of thin-walled parts processing error sources, the thin-walled parts machining process is divided into two processes of cutting and clamping, and error models of the two processes are established. Finally, taking a simplified model of Thin-walled parts as an example, the effect of the errors adjustment method is verified by the result.
\end{abstract}

\section{Introduction}

Aeronautical complex thin-walled parts which have the characteristics of light weight and tight structure are widely used in aviation industry. However, at the same time, the material is difficult to cut, the rigidity is very low, and the product quality is affected by multiply operations and error sources. In order to produce high quality components to meet the requirements of modern design, we carrying out the researches about aeronautical thin-walled parts processing error modeling and error compensation technology has application value. For thin-walled parts processing error modeling problem, given the fixture-workpiece-tool system geometric error model and the integrated error model consideration of geometry, physics error sources is built on this thesis. Based on the analysis of thin-walled parts processing error sources, the thin-walled parts machining process is divided into two processes of cutting and clamping, and error models of the two processes are established as shown in Fig.1:

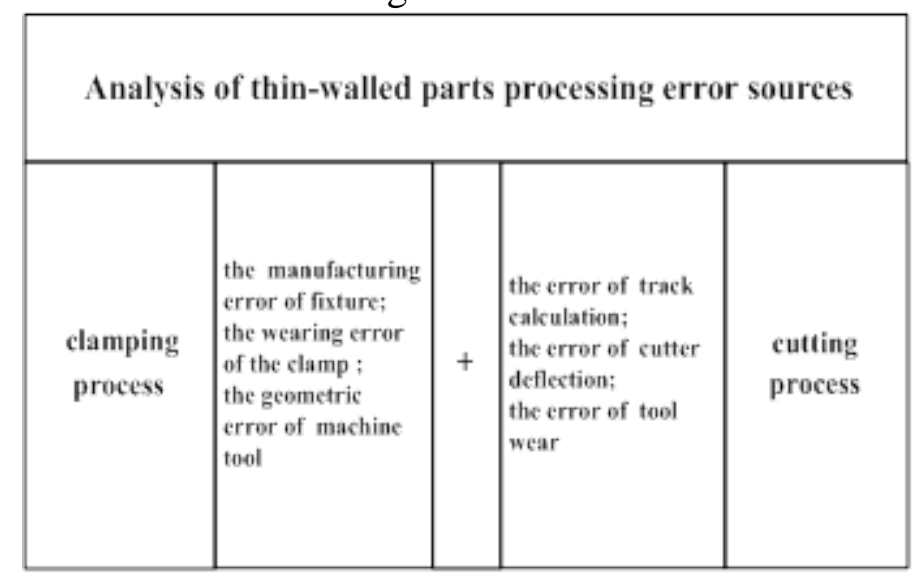

Fig.1. Analysis of Error Source

1.Firstly, in the clamping process, when the source of geometric errors introducing during clamping thin-walled parts, the fixture-workpiece-tool system error model is established;

2.Secondly, in the cutting process, innovatively consider plenty kinds of geometric and physical error sources,thin-walled parts processing integrated error model is built by using bicubic B-spline interpolation. 


\section{An Adjustment Method of Clamping Process based on Wavelet Real-Time Denoising and MEPC}

Using the method of Bias state space to establish a quality fluctuation model of the machining process that can be expressed as:

$$
\left\{\begin{array}{l}
x_{t}=\alpha+\beta c_{t-1}+\varepsilon_{t}, \varepsilon_{t} \sim N[0, W] \\
y_{t}=x_{t}+v_{t}, v_{t} \sim N[0, V]
\end{array}\right.
$$

MEPC adjustment is used to the (1) expression, and by using recursion, it can be known, after processing the $t$ product, the mean of machining process quality characteristic fluctuation is:

$$
x_{t}=(1-\omega)^{t-1}\left(y_{1}-\tau\right)+\sum_{i=0}^{t-2}(1-\omega)^{i}\left(\varepsilon_{t-i}-\varepsilon_{t-i-1}\right)-\sum_{i=0}^{t-2} \omega(1-\omega)^{i} v_{t-i-1}
$$

Consider the presence of noise and show the error mode [1] in the form of state space as follows:

$$
\left\{\begin{array}{l}
\Delta X_{t s}^{f s^{0}}=\Gamma * \Delta X_{t T}^{G}-\Gamma *\left[\hat{G}^{+} *\left(-D * \Delta x_{t d}+N * \Delta r_{t f}\right)\right]+\varepsilon_{t} \\
\Delta Y_{t f s}^{f s^{0}}=\Delta X_{t f s}^{f s^{0}}+v_{t}
\end{array}\right.
$$

Using the concept of equivalent error [2] to establish MEWMA adjustment model:

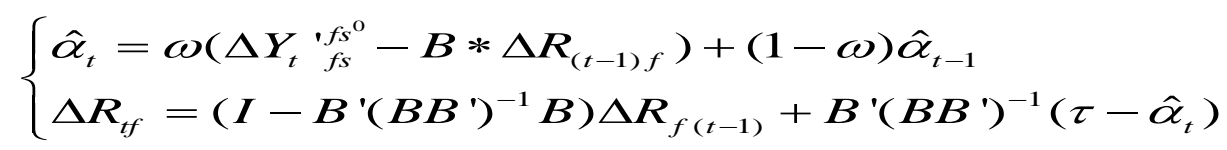

According YIBO JIAO [3] and other evaluations of the proposed control law, the proposed method with the traditional MEWMA controller to adjust the results are compared by the (5) expression:

$$
\left\{\begin{array}{l}
\lambda=\frac{\left\|\left[\mu_{\mathrm{X}}^{1 \cdots N}\right]\right\|-\left\|\left[\mu_{\mathrm{X}^{\prime}}^{1 \cdots N}\right]\right\|}{\left\|\left[\mu_{X}^{1 \cdots N}\right]\right\|} \times 100 \% \\
\eta=\frac{\left\|\left[\sum_{X}^{1 \cdots \cdots}\right]\right\|-\left\|\left[\sum_{X^{\prime}}^{1 \cdots N}\right]\right\|}{\left\|\left[\sum_{X}^{1 \cdots N}\right]\right\|} \times 100 \%
\end{array}\right.
$$

If $\lambda>0, \eta>0$, it this new method is superior to the traditional one of adjusting MEWMA.

\section{Test Results}

Now take of the Engine blade processing working procedure as an example,This article will simplify the body-surface structure to thin-walled parts, conduct the milling operations at the tip of the plane.

Calculate performance parameters $\lambda, \eta$, and comparison of the effect of two methods are shown in Table 1:

Table.1. performance parameters $\lambda, \quad \eta$

\begin{tabular}{|c|c|c|c|c|}
\hline & $\begin{array}{c}\text { The traditional } \\
\text { method }\end{array}$ & The new method & \multicolumn{2}{|c|}{$\begin{array}{c}\text { Calculation of performance } \\
\text { parameters }\end{array}$} \\
\hline The average norm & 0.2307 & 0.2107 & $\lambda$ & $8.65 \%$ \\
\hline The variance norm & 6.8597 & 6.6396 & $\eta$ & $3.21 \%$ \\
\hline
\end{tabular}


Use of the proposed method makes the mean of characteristic pose error vector and the norm of variance than the traditional MEWMA adjustment method decreased by $8.65 \%$, 3.21\%, which is better than the traditional method of adjusting MEWMA.

\section{The Machining Error Compensation Method for Comprehensive Error Sources of Thin-walled Parts}

When control points $V_{i j}(i=0,1, \ldots, n ; j=0,1, \ldots, m)$ has been known, the following parameters surfaces are the B-spline surfaces as follows:

$$
r(u, w)=\sum_{i=0}^{n} \sum_{j=0}^{m} N_{i, n}(u) N_{j, m}(w) V_{i j}, \quad 0 \leq u \leq 1,0 \leq w \leq 1
$$

And, $\quad r(u, w)=U B V B^{T} W^{T}$

$$
\text { In this type: } \quad U=\left[1, u, u^{2}, u^{3}\right], W=\left[1, w, w^{2}, w^{3}\right] ; V=\left[\begin{array}{llll}
V_{0,0} & V_{0,1} & V_{0,2} & V_{0,3} \\
V_{1,0} & V_{1,1} & V_{1,2} & V_{1,3} \\
V_{2,0} & V_{2,1} & V_{2,2} & V_{2,3} \\
V_{3,0} & V_{3,1} & V_{3,2} & V_{3,3}
\end{array}\right], \mathrm{B}=\frac{1}{6}\left[\begin{array}{cccc}
1 & 4 & 1 & 0 \\
-3 & 0 & 3 & 0 \\
3 & -6 & 3 & 0 \\
-1 & 3 & -3 & 1
\end{array}\right]
$$

According to the anti deformation compensation principle[4], the relationship of the modified cutter location, name of cutter location location location and the machining error of the three can be expressed as:

$$
X_{c i}=X_{d i}+U_{i}
$$

After considering the impact of iterations between force and deformation of the workpiece to establish a flexible compensation model.Between the cutting force model and finite element model, through iterative calculations to obtain the i-th final compensation amount cutter sites, then correct nominal toolpaths, and move on to the next site to continue calculating the knife until completing the compensation and correction of all the cutter location data.

\section{Test Results}

In the sheet for the processing of objects, we design to use thin-walled parts machining error compensation method experiments to verify the effectiveness of the compensation method.With small depth of cut small feed repeatedly finishing and eventually get two pieces of the same size, which are $60 \mathrm{~mm} * 40 \mathrm{~mm} * 2.5 \mathrm{~mm}$ of A and B thin workpieces as Figure. 2 and Figure. 3 shows:

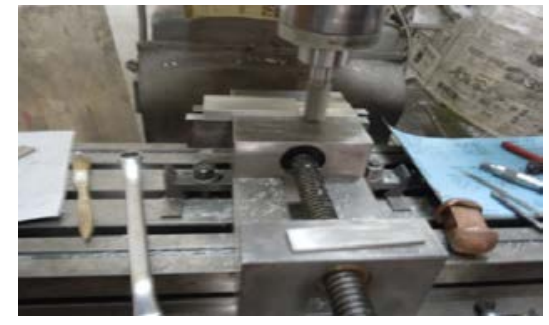

Fig.2. The processing of thin-walled parts

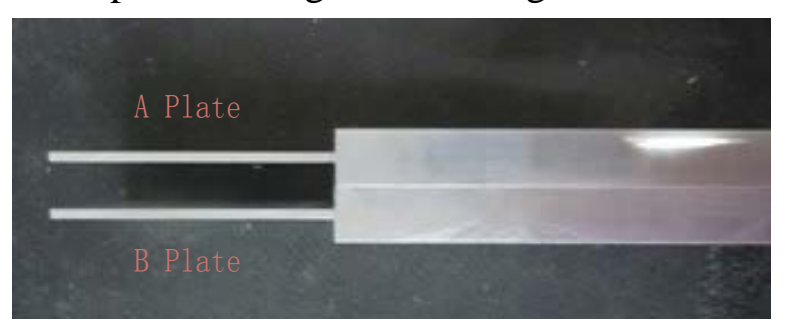

Fig.3. Plate A and Plate B

Firstly,regarding the A plate as a historical workpiece, the last layer of the A plate is cutting. Access the historical processing error data, and use the algorithms to construct bicubic B-spline interpolation surface.Solve the error prediction model to obtain the surface of each thin-walled parts relations between knife sites and error distribution.

Secondly, using a mechanical method that Budak[5] proposed fast calibration milling cutting force coefficient to calibrate milling force coefficient. The simulation analysis on the stress of thin plate workpieces is finished by using the finite element software Abaqus as shown in Figure.4: 
Finally, based on a comprehensive process of cutting thin workpiece machining error model and error compensation scheme to compensate for the machining error on B plate under the same processing conditions for processing. Measure the error distribution and verify the effectiveness of the method as shown in Figure.5.

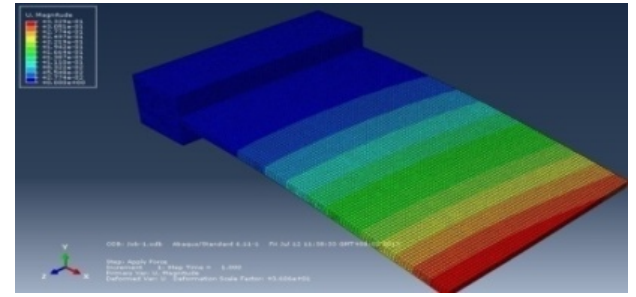

Fig.4. Finite element model

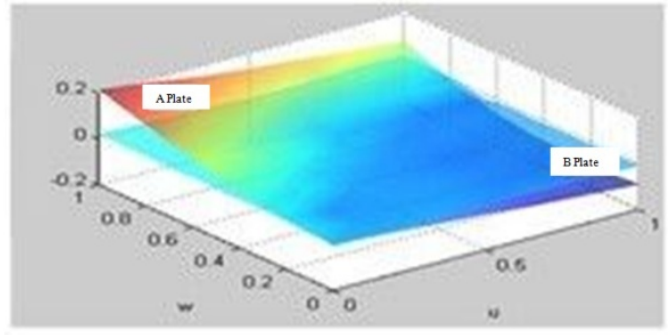

Fig.5. Machining error compensation effect

\section{Conclusion}

This paper studies the aerospace thin-walled parts processing error modeling techniques, error adjustment and compensation methods. Divide thin-walled workpieces manufacturing process into two processes(clamping and cutting),establish a error model, and propose a adjustment of the corresponding process error compensation method. For the relevant numerical example and experimental verification, the results show that the proposed method can effectively improve the precision machining of thin-walled parts.

\section{Acknowledgement}

In this paper, the research was sponsored by the Xi'an Science and Technology Project (Project No. CXY1313 and No. CXY1338(5)).

\section{References}

[1] Wang Xu-hui. Effect of measurement system Error on process quality[D].Tianjin:Tianjin University,2009

[2] Gao Jing, Li Chao -wei. Simulation on the seeker signal wavelets denoising based On parameters optimization[J[,Aero Weaponry,2010,(5):48-54

[3] YIBO JIAO, DRAGAN DJURDJANOVIC. “Joint allocation of measurement points and controllable tooling machines in multistage manufacturing processes", IIE Transactions, 2010(42):703-720.

[4] Liu Ying,Li Yan. Application of a multi-algorithm fusion real time filter in FOGs[J].Acta Photonica Sinica,2010,39(6):1116-1119

[5] Budak E, Altintas Y. Armarego E J A. Prediction of milling force coefficientsfrom orthogonal cutting data[J]. Transactions of the ASME Journal of Manufacturing Science and Engineering, 1996, 118(2):216-224 